\title{
PERMEABILIDADE AO AR DA CAMA DE SEMEADURA DO SOLO EM SISTEMA SEMEADURA DIRETA
}

\author{
Osvaldo Guedes Filho(1)*, Alvaro Pires da Silva ${ }^{(2)}$, Neyde Fabíola Balarezo Giarola ${ }^{(3)}$ e Cássio \\ Antônio Tormena ${ }^{(4)}$ \\ (1) Universidade Federal do Paraná, Campus Avançado de Jandaia do Sul, Jandaia do Sul, Paraná, Brasil. \\ (2) Universidade de São Paulo, Escola Superior de Agricultura Luiz de Queiroz, Departamento de Ciência do Solo, Piracicaba, São \\ Paulo, Brasil. \\ (3) Universidade Estadual de Ponta Grossa, Departamento de Ciências do Solo e Engenharia Agrícola, Ponta Grossa, Paraná, Brasil. \\ (4) Universidade Estadual de Maringá, Departamento de Agronomia, Maringá, Paraná, Brasil. \\ * Autor correspondente. \\ E-mail: osvaldoguedes@ufpr.br
}

\section{RESUMO}

A permeabilidade ao ar pode ser utilizada como indicador da qualidade física do solo. $O$ objetivo deste trabalho foi determinar a permeabilidade ao ar e os índices de continuidade de poros para a cama de semeadura em um Latossolo Vermelho distrófico cultivado no sistema semeadura direta e submetido à escarificação mecânica e escarificação biológica, utilizando a cultura do nabo forrageiro. $O$ estudo foi conduzido em área experimental da Universidade Estadual de Ponta Grossa, Estado do Paraná. Os tratamentos implantados foram: sistema semeadura direta por 18 anos consecutivos (SD); semeadura direta submetido à escarificação mecânica (SDE); e semeadura direta submetido à escarificação biológica por meio da cultura do nabo forrageiro (SDNF). $O$ delineamento experimental utilizado foi em blocos casualizados com quatro repetições. As amostragens de solo foram feitas aos seis e 18 meses após a implantação dos tratamentos, correspondentes às semeaduras das culturas do milho (outubro de 2009) e da soja (novembro de 2010), respectivamente, nas camadas de 0,00-0,05 e 0,05-0,10 m de profundidade. A permeabilidade ao ar foi determinada pelo permeâmetro de carga constante nos potenciais mátricos $-6,-10,-30$ e $-100 \mathrm{kPa}$. Foram definidos os seguintes índices de continuidade de poros: índice $\mathrm{N}$, índice $K 1$ e volume de poros bloqueados; e a porosidade de aeração. Os resultados foram submetidos à análise de variância e, quando significativos, as médias dos tratamentos foram comparadas pelo teste Tukey $(p<0,05)$. Para a camada de 0,00-0,05 m, a permeabilidade ao ar e o índice $K 1$ no SDNF no potencial mátrico de $-6 \mathrm{kPa}$ foram significativamente maiores que em SD e SDE. $O$ índice N, o volume de poros 
bloqueados e a porosidade de aeração não apresentaram diferenças significativas entre os tratamentos em ambas as profundidades. Os efeitos benéficos da escarificação biológica na permeabilidade do solo ao ar e no índice de continuidade de poros $K 1$ persistiram aos 18 meses após sua aplicação. A escarificação mecânica resultou em maior continuidade do sistema poroso do solo, avaliado pelo índice $K 1$, com persistência desse efeito aos 18 meses após sua aplicação.

Palavras-chave: porosidade de aeração, índice N, porosidade bloqueada, índice $K 1$.

\section{ABSTRACT: AIR PERMEABILITY OF THE SOIL SEEDBED UNDER A NO-TILLAGE SYSTEM}

Air permeability may be used as a soil physical quality indicator. The objective of this study was to determine the air permeability and pore continuity index for the seedbed of an Oxisol cultivated under a no-tillage system that underwent mechanical chiseling and biological promotion of pore formation using the forage radish crop. The study was carried out in an experimental area of the Universidade Estadual de Ponta Grossa (Ponta Grossa State University), Parana, Brazil. The treatments were 18 consecutive years under no tillage (SD); no-tillage under mechanical chiseling (SDE); and no-tillage under biological promotion of pore formation by a forage radish cover crop (SDNF). A randomized complete block experimental design was used with four replications. Soil samples were taken at the 0.00-0.05 and 0.05-0.10 $\mathrm{m}$ soil depths at 6 and 18 months after setting up the treatments, corresponding to maize (October 2009) and soybean (November 2010) planting, respectively. Air permeability was determined with a constant head permeameter at four matric potentials: -6, -10, -30, and -100 kPa. The following pore continuity indices were determined: $N$ index, K1 index, and blocked porosity; and air-filled porosity. The results were subjected to analysis of variance and, when significant, the means were compared by the Tukey test $(p<0.05)$. For the 0.00-0.05 $\mathrm{m}$ soil depth, the air permeability and K1 index in SDNF at the - 6 kPa matric potential were significantly higher than in SD and SDE. The N index, blocked porosity and air-filled porosity did not differ statistically among treatments for either depth. The beneficial effects of biological promotion of pore formation on air permeability and on the soil pore continuity index (K1) continued at 18 months after application. Mechanical chiseling resulted in greater continuity of the soil pore system (K1), and this effect persisted at 18 months after application.

Keywords: air-filled porosity, $N$ index, blocked porosity, $K 1$ index.

\section{INTRODUÇÃO}

A qualidade da cama de semeadura é fundamental para os processos de germinação e crescimento inicial das culturas, pois seu arranjo estrutural tem impacto direto na temperatura, no conteúdo de água, na disponibilidade de oxigênio e na resistência do solo à penetração, estabelecendo um ambiente físico crucial para o desenvolvimento e estabelecimento das culturas (Atkinson et al., 2007, 2009).

A cama de semeadura é definida para sistemas de manejo que envolvem o preparo do solo, como uma camada superficial solta e rasa (Braunack e Dexter, 1989; Hakansson et al., 2002). Porém, para sistemas de manejo conservacionistas do solo, como no caso do sistema semeadura direta, ela é caracterizada como a camada superficial do solo mobilizada apenas pelos discos ou hastes sulcadoras utilizadas durante a semeadura e fortemente influenciada pela atividade biológica dos diferentes sistemas radiculares das espécies utilizadas na rotação de culturas (Guedes Filho et al., 2013). O sistema semeadura direta é um dos mais importantes sistemas conservacionistas de manejo do solo, sendo cultivado em uma área de aproximadamente 117 milhões de hectares no mundo, dos quais cerca de 32 milhões de hectares estão no Brasil (Febrapdp, 2014). Todavia, a ausência de revolvimento e principalmente o intenso tráfego de máquinas são considerados os principais responsáveis pela compactação superficial do solo sob semeadura direta (Reichert et al., 2009).

A escarificação mecânica tem sido utilizada como forma de aliviar a compactação superficial dos solos manejados sob sistema semeadura direta (Evans et al., 1996; Câmara e Klein, 2005). O principal objetivo dessa prática é romper a camada compactada de forma a aumentar a porosidade, diminuir a densidade e aumentar a infiltração e a disponibilidade de água no solo. No entanto, na maioria dos estudos, observou-se que a escarificação mecânica do solo tende a ser uma prática cujos efeitos são de caráter temporário (Secco e Reinert, 1997; Vieira e Klein, 2007; Nicoloso et al., 2008; Nunes et al., 2014). Entretanto, plantas com sistema radicular profundo e agressivo, introduzidas em rotação de culturas, têm sido uma estratégia utilizada para recuperação estrutural do solo e alívio dos problemas de compactação em semeadura direta (Unger e Kaspar, 1994). 
As principais culturas utilizadas com a finalidade de promover a recuperação estrutural do solo são alfafa (Meek et al., 1990), aveia (Nicoloso et al., 2008), canola (Williams e Weil, 2004), colza (Chen e Weil, 2010), crotalária (Abreu et al., 2004; Reinert et al., 2008), braquiária (Calonego et al., 2011) e nabo forrageiro (Williams e Weil, 2004; Kubota et al., 2005; Nicoloso et al., 2008; Reinert et al., 2008; Chen e Weil, 2010; Guedes Filho et al., 2013). Angers e Caron (1998) relataram que, com o cultivo de plantas de sistema radicular agressivo, ocorre a compressão do solo em torno da raiz, o que promove o alargamento dos poros existentes e a criação de novos poros. Aliado a isso, o efeito biodrilling ou bioperfuração, descrito por Cresswell e Kirkegaard (1995), propicia o aumento no tamanho dos macroporos do solo com consequente aumento da infiltração de água e na permeabilidade ao ar, além de criar caminhos preferenciais para o desenvolvimento radicular da cultura subsequente.

A permeabilidade do solo ao ar $(K a)$ é uma propriedade que descreve a habilidade do meio em prover a troca de gases entre o solo e a atmosfera, determinando a disponibilidade de oxigênio para o sistema radicular das plantas e interferindo diretamente no seu desenvolvimento. Assim, a quantificação de propriedades relacionadas com a capacidade do solo em transmitir $\mathrm{O}_{2}$ até as raízes é fundamental para a avaliação de sua qualidade física (Silva et al., 2009).

A permeabilidade ao ar também pode ser usada para inferir sobre as características de poros do solo, pois sofre influência do conteúdo de água no solo, apresentando seu máximo valor em solo seco e o mínimo quando esse está na saturação (Schjønning et al., 1999). Dessa forma, ela é fortemente influenciada pela porosidade livre de água, uma vez que uma redução no conteúdo de água implica em aumento da porosidade de aeração. Mudanças na geometria dos poros foram avaliadas pela equação de Kozeny-Carman, que relaciona $K a$ e porosidade de aeração, na forma linearizada descrita por Ball et al. (1988). Nessa equação, o slope da equação $(N)$ é considerado um índice de continuidade dos poros, refletindo a elevação na $K a$ com o aumento na porosidade de aeração ou o decréscimo na tortuosidade dos poros e da área superficial para o fluxo com o aumento da fração de poros disponíveis. Rodrigues et al. (2011) determinaram o índice $\mathrm{N}$ em semeadura direta e preparo convencional e observaram maior valor de $\mathrm{N}$ para a camada de $0,10-0,20 \mathrm{~m}$ do que de 0,00-0,10 m em sistema semeadura direta. Eles concluíram que a semeadura direta tende a preservar a continuidade de poros em subsuperfície. Os resultados de índice N encontrados por Roseberg e McCoy (1990) e Ball et al. (1988) confirmaram essa tendência do sistema semeadura direta.

O conhecimento da quantidade de ar disponível no solo em relação às práticas de manejo pode auxiliar os agricultores e pesquisadores a planejar com eficiência os sistemas de produção agrícola (Rodrigues et al., 2011). A hipótese estabelecida neste estudo foi que tanto a escarificação mecânica quanto a escarificação biológica promoveriam melhoria da permeabilidade ao ar da cama de semeadura em solo cultivado em sistema semeadura direta. O objetivo foi determinar a permeabilidade ao ar e índices de continuidade de poros para a cama de semeadura em um Latossolo Vermelho distrófico cultivado no sistema semeadura direta e submetido à escarificação mecânica e à biológica com a cultura do nabo forrageiro (Raphanus sativus L.).

\section{MATERIAL E MÉTODOS}

O estudo foi conduzido em área localizada na Fazenda Experimental "Capão da Onça”, pertencente à Universidade Estadual de Ponta Grossa (UEPG), localizada no município de Ponta Grossa, Estado do Paraná. As coordenadas geográficas da área são $25^{\circ} 05^{\prime} 52^{\prime \prime} \mathrm{S}$ e $50^{\circ} 02^{\prime} 43^{\prime \prime} \mathrm{O}$. O solo da área é um Latossolo Vermelho distrófico típico, de classe textural argiloarenosa (Embrapa, 2006) com declividade inferior a $1 \%$ e altitude de $1.080 \mathrm{~m}$. O clima da região é classificado, segundo Köppen, como mesotérmico subtropical úmido do tipo $\mathrm{Cfb}$, com precipitação pluvial média anual de $1.545 \mathrm{~mm}$ e temperatura média anual de $18,7^{\circ} \mathrm{C}$ (Iapar, 2000).

A área experimental ( 0,7 ha) é manejada com sistema semeadura direta há 18 anos e submetida à rotação de culturas com milho (Zea mays L.) e soja (Glycine $\max$ L.), na primavera/verão, e trigo (Triticum aestivum L.) e consórcio aveia-preta (Avena strigosa Schreb) + ervilhaca (Vicia sativa L.), no outono/inverno, resultando na seguinte sequência: milho - aveia-preta + ervilhaca - soja - trigo - milho. $\mathrm{O}$ experimento foi instalado em maio de 2009 , com três tratamentos: semeadura direta escarificada (SDE), semeadura direta cultivada com nabo forrageiro (SDNF) e semeadura direta (SD). A escarificação mecânica e o cultivo da planta nabo forrageiro (Raphanus sativus L.) foram introduzidos na área de cultivo como agentes de descompactação mecânica e biológica do solo, respectivamente.

O delineamento experimental utilizado foi de blocos casualizados com quatro repetições, resultando em 12 parcelas com dimensões de $9 \times 50 \mathrm{~m}$ cada. As parcelas com SDE foram escarificadas em maio de 2009 com um subsolador tipo Asa laser da Jumbo Matic ${ }^{\circledR}$, dois dias antes da semeadura da cultura de inverno, até a profundidade de $0,25 \mathrm{~m}$. O subsolador possuía cinco hastes, sendo três na barra dianteira e duas na traseira, de formato parabólico e ponteira em cinzel, com espaçamento entre hastes de $0,20 \mathrm{~m}$ e ângulo de ataque de $22^{\circ}$. Nas parcelas 
SDNF, o nabo forrageiro foi semeado com densidade de 22 sementes $\mathrm{m}^{-1}$ e espaçamento de $0,17 \mathrm{~m}$ na entrelinha. As parcelas SD foram cultivadas com aveia + ervilhaca, seguindo a sequência de rotação de culturas estabelecidas para o outono/inverno, bem como as parcelas SDE, após a operação da escarificação mecânica. Para semeadura das culturas do milho e da soja, foi utilizada semeadora com discos para colocação das sementes a $0,04 \mathrm{~m}$ e com hastes sulcadoras para deposição do adubo a $0,10 \mathrm{~m}$, mobilizando o solo somente nas linhas de semeadura.

As amostragens foram realizadas em outubro de 2009 e novembro de 2010, respectivamente durante a fase de cama de semeadura das culturas do milho e da soja. A primeira avaliação ocorreu aos seis meses e a segunda aos 18 meses, após a implantação do experimento. Foram coletadas 10 amostras indeformadas de solo (anéis volumétricos de $0,05 \times 0,05 \mathrm{~m}$ ) por parcela, em pontos selecionados aleatoriamente, nas camadas de 0,00-0,05 e 0,05-0,10 $\mathrm{m}$ de profundidade. As amostras foram coletadas na linha de semeadura da cultura aos 15 dias após a semeadura. Para a coleta de solo, foi utilizado um amostrador eletromecânico que permitiu a retirada das amostras de solo sem aplicação de golpes e com deslocamento do eixo a uma velocidade constante de $2 \mathrm{~mm} \mathrm{~s}^{-1}$ (Figueiredo, 2010). As amostras foram embaladas e acondicionadas em recipientes adequados para o transporte e enviadas ao laboratório, onde foram mantidas em temperatura de aproximadamente $5{ }^{\circ} \mathrm{C}$ até serem processadas.

Para determinar a permeabilidade ao ar $(K a)$, as amostras foram divididas em quatro potenciais mátricos: - 6 ; -10; -30; e -100 kPa, em mesa de tensão e placas porosas em câmaras de pressão. Após atingir o equilíbrio, as amostras foram pesadas para cálculo da umidade do solo no referido potencial mátrico e em seguida realizou-se a definição da permeabilidade do solo ao ar por meio do permeâmetro de carga constante de ar desenvolvido por Figueiredo (2010). O princípio de funcionamento desse aparelho consiste da utilização de amostra de solo em anel volumétrico e com livre fluxo de gases em suas extremidades, sendo submetida a incrementos sucessivos e constantes de taxas de fluxo de ar para gerar distintos gradientes de pressão. A partir da Lei de Darcy, a densidade de fluxo de ar (q) pode ser descrita como:

$$
\mathrm{q}=-\frac{K_{a}}{\mathrm{r}}\left(\frac{\mathrm{dP}}{\mathrm{dZ}}+\rho \mathrm{g}\right)
$$

sendo $\mathrm{q}=[\mathrm{L} / \mathrm{T}], K a\left[\mathrm{~L}^{2}\right], \mathrm{P}=$ a pressão de $\operatorname{ar}\left[\mathrm{M} / \mathrm{LT}^{2}\right]$, $\mathrm{z}=$ a distância $[\mathrm{L}]$ na direção do movimento de ar no sistema poroso, $\mathrm{n}=$ a viscosidade do ar $[\mathrm{M} / \mathrm{LT}]$ $\left(18,1 \times 10^{-6} \mathrm{~kg} \mathrm{~m}^{-1} \mathrm{~s}^{-1}\right.$ a $\left.20^{\circ} \mathrm{C}\right), \rho=$ a densidade do ar $\left[\mathrm{M} / \mathrm{L}^{3}\right]$ e $\mathrm{g}=\mathrm{a}$ aceleração da gravidade $\left[\mathrm{L} / \mathrm{T}^{2}\right]$.

A densidade de fluxo corresponde à vazão de ar (Q) $\left[\mathrm{L}^{3} / \mathrm{T}\right]$ por área perpendicular ao movimento do fluido $\left(\mathrm{A}_{\mathrm{s}}\right)\left[\mathrm{L}^{2}\right]$ e, considerando como desprezível a influência da densidade do ar (Springer et al., 1998), a equação 1 foi utilizada para as estimativas da $K_{a}$ :

$$
\mathrm{Ka}=\frac{\mathrm{Q}_{\mathrm{n}}}{\mathrm{A}_{\mathrm{S}}}\left(\frac{\mathrm{dP}}{\mathrm{dZ}}\right)
$$

Para avaliar a continuidade de poros, os valores de $K a$ e porosidade de aeração $\left(\varepsilon_{a}\right)$ foram relacionados usando a equação de Kozeny-Carman, conforme Ahuja et al. (1984):

$$
K a=\mathrm{M \varepsilon}_{\mathrm{a}}{ }^{\mathrm{N}}
$$

em que $\mathrm{M}$ e $\mathrm{N}$ são constantes empíricas. Os autores consideraram o expoente $\mathrm{N}$ como um índice de continuidade de poros, que reflete o aumento da $K a$ com a elevação da $\varepsilon_{a}$ ou o decréscimo da tortuosidade dos poros e área superficial com o aumento da fração de poros disponíveis para o fluxo. A relação de $K a$ e $\varepsilon_{a}$ foi ajustada para uma forma linearizada da equação 3:

$$
\log K_{a}=\log \mathrm{M}+\mathrm{N} \log \varepsilon_{a}
$$

Da relação linearizada na equação 4 , pode-se obter três informações indicadas na figura 1: o slope $(\mathrm{N})$; o intercepto com o eixo log $K a$, em que $\log \varepsilon_{a}=0(\mathrm{M})$; e o intercepto com o eixo $\log \varepsilon_{a}$, em que $\log K a=0\left(\varepsilon_{\mathrm{b}}\right)$. Este intercepto com o eixo log $\varepsilon_{a}$ é denominado de conteúdo de ar limitante e foi proposto por Ball et al. (1988) e Schjønning et al. (2002), como a quantidade de poros de aeração bloqueados no solo que não faz parte do transporte convectivo do ar. Desse modo, $\varepsilon_{\mathrm{b}}$ é o valor de $\varepsilon_{\mathrm{a}}$ abaixo do qual o fluxo de ar por meio do solo cessa em razão da descontinuidade na rede de poros de aeração e pode ser obtido da equação 4 pela 5 :

$$
\varepsilon_{\mathrm{b}}=10^{(-\log \mathrm{M}) / \mathrm{N}}
$$

$\mathrm{O}$ índice de continuidade de poros $K_{1}$ proposto por Groenevelt et al. (1984) também foi calculado com base na relação entre $K a$ e $\varepsilon_{a}$ :

$$
K_{1}=\frac{K_{a}}{\varepsilon_{\mathrm{a}}}
$$

A porosidade de aeração $\left(\varepsilon_{a}\right)$ foi calculada de acordo com a equação 7 :

$$
\varepsilon_{\mathrm{a}}=\mathrm{PT}-\theta
$$

em que $\varepsilon_{a}$ é a porosidade de aeração, $\mathrm{em} \mathrm{m}^{3} \mathrm{~m}^{-3}$; e $\theta$, a umidade volumétrica, em $\mathrm{m}^{3} \mathrm{~m}^{-3}$.

Os dados de permeabilidade ao ar foram submetidos ao teste de normalidade, de acordo com Shapiro e Wilk (1965). A análise de variância foi usada para avaliar os efeitos dos tratamentos e as épocas de coleta dentro de cada profundidade. A interação entre épocas de amostragem foi testada e quando houve interação significativa foi feito o seu desdobramento. As médias foram comparadas pelo teste Tukey a $5 \%$. 
Todas as análises estatísticas foram desenvolvidas no software SAS (SAS Institute, 2002).

\section{RESULTADOS E DISCUSSÃO}

Os dados de permeabilidade ao ar não apresentaram distribuição normal; em razão disso, seus valores foram transformados para a forma logarítmica com vistas a obter distribuição mais próxima possível da normalidade. Os valores médios de log $K a$ para todos os potenciais utilizados estão apresentados no quadro 1 .

A análise de variância revelou interação significativa entre tratamentos e épocas de amostragem para a permeabilidade ao ar. A $K a$ apresentou efeito significativo entre os tratamentos somente para o potencial mátrico de $-6 \mathrm{kPa}$, na camada de 0,00-0,05 m, na primeira época avaliada (2009); e no potencial de $-30 \mathrm{kPa}$, na camada de 0,00-0,05 m, na segunda época avaliada (2010). No potencial mátrico equivalente a $-6 \mathrm{kPa}$, o $\mathrm{SDNF}$ teve permeabilidade ao ar maior que SDE e SD, enquanto no potencial de $-30 \mathrm{kPa}$ os valores de $K a$ em SDNF e SDE foram maiores que o SD. Esses resultados apontam para maior eficiência do cultivo do nabo forrageiro em relação à permeabilidade do solo ao ar, como também relatado por Cresswell e Kirkegaard (1995). Provavelmente, o sistema radicular mais resistente e agressivo do nabo forrageiro tenha promovido melhoria da estrutura do solo, resultando em maior permeabilidade aos 18 meses após seu cultivo. Resultados semelhantes aos deste estudo foram obtidos por

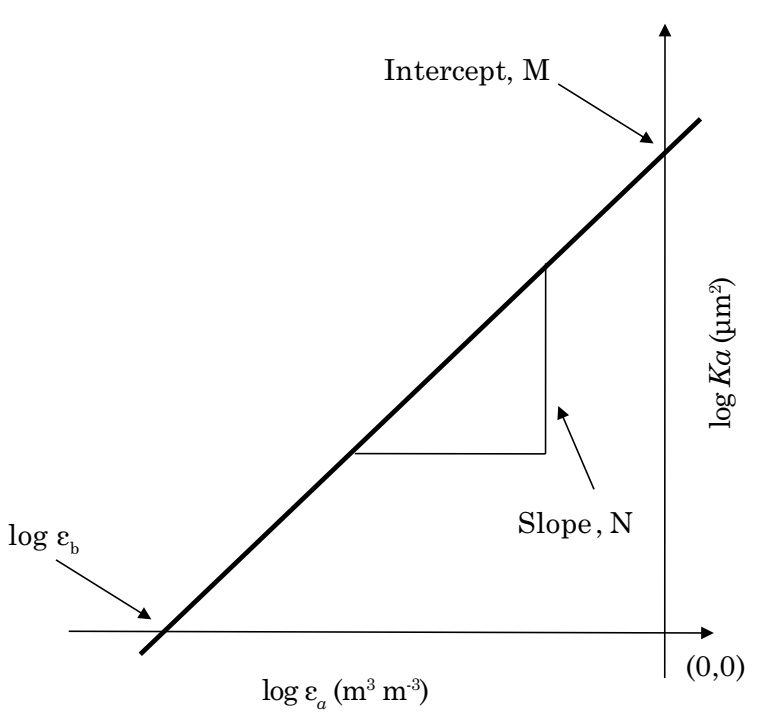

Figura 1. Principais características da relação log (porosidade de aeração, $\varepsilon_{a}$ ) - log (permeabilidade ao ar $\mathrm{Ka}$ ); slope $\mathrm{N}$, intercepto com o eixo log $(K a), M$ é o limite da porosidade de aeração, $\varepsilon_{\mathrm{b}}$.
Silveira Junior et al. (2012), quando avaliaram a permeabilidade ao ar para o mesmo experimento; e por Nicoloso et al. (2008), os quais destacaram a eficiência da escarificação biológica em aumentar a macroporosidade e reduzir a resistência do solo à penetração em um Latossolo Vermelho.

$\mathrm{Na}$ avaliação dos tratamentos entre as épocas, para a camada de 0,00-0,05 m, apenas nos potenciais mátricos de - 6 e - $10 \mathrm{kPa}$ a $K a$ foi maior em SDE e SD em 2010, comparado com 2009 (Quadro 1). Esse resultado indica que houve melhoria do sistema poroso do solo de 2009 para 2010, em SDE e SD, mas não em SDNF. Isso possivelmente resultou em ausência de diferenças estatísticas entre tratamentos para o ano de 2010, no potencial mátrico de $-6 \mathrm{kPa}$. Para os demais potenciais na camada de 0,00-0,05 $\mathrm{m}$ e para todos os potenciais mátricos na camada de 0,05-0,10 m, não se verificaram diferenças significativas entre 2009 e 2010 para cada tratamento individualmente.

Houve ocorrência de $K a$ igual zero para os tratamentos SDE e SD no potencial mátrico de $-6 \mathrm{kPa}$ na camada de $0,00-0,05 \mathrm{~m}$ e para todos os tratamentos na camada de $0,05-0,10 \mathrm{~m}$, nos

Quadro 1. Valores médios de permeabilidade ao ar $(\log K a)$ para a cama de semeadura das culturas do milho (2009) e soja (2010) para as camadas de 0,00-0,05 e 0,05-0,10 $\mathrm{m}$ de profundidade nos potenciais mátricos de $-6,-10,-30$ e $-100 \mathrm{kPa}$

\begin{tabular}{lcccc}
\hline \multirow{2}{*}{ Tratamento } & \multicolumn{2}{c}{$\mathbf{0 , 0 0 - 0 , 0 5} \mathbf{~ m}$} & $\mathbf{0 , 0 5 - 0 , 1 0 ~} \mathbf{~}$ \\
\cline { 2 - 5 } & $\mathbf{2 0 0 9}$ & $\mathbf{2 0 1 0}$ & $\mathbf{2 0 0 9}$ & $\mathbf{2 0 1 0}$ \\
\hline \multirow{5}{*}{$-6 \mathrm{kPa} K a\left(\mu \mathrm{m}^{2}\right)$} \\
SDE & $-0,58 \mathrm{bB}$ & $0,12 \mathrm{aA}$ & $-0,89 \mathrm{aA}$ & $-1,22 \mathrm{aA}$ \\
SD & $-0,53 \mathrm{bB}$ & $0,57 \mathrm{aA}$ & $-1,19 \mathrm{aA}$ & $-0,20 \mathrm{aA}$ \\
SDNF & $0,09 \mathrm{aA}$ & $0,47 \mathrm{aA}$ & $-1,19 \mathrm{aA}$ & $-0,98 \mathrm{aA}$ \\
& & $-10 \mathrm{kPa}$ & & \\
SDE & $-0,33 \mathrm{aB}$ & $0,92 \mathrm{aA}$ & $-0,49 \mathrm{aA}$ & $-0,79 \mathrm{aA}$ \\
SD & $0,28 \mathrm{aB}$ & $0,68 \mathrm{aA}$ & $-0,98 \mathrm{aA}$ & $-0,04 \mathrm{aA}$ \\
SDNF & $0,15 \mathrm{aA}$ & $0,83 \mathrm{aA}$ & $-0,69 \mathrm{aA}$ & $-0,49 \mathrm{aA}$ \\
& & $-30 \mathrm{kPa}$ & & \\
SDE & $0,33 \mathrm{aA}$ & $0,86 \mathrm{aA}$ & $0,03 \mathrm{aA}$ & $-0,37 \mathrm{aA}$ \\
SD & $0,37 \mathrm{aA}$ & $0,79 \mathrm{bA}$ & $-0,44 \mathrm{aA}$ & $-0,57 \mathrm{aA}$ \\
SDNF & $0,42 \mathrm{aA}$ & $1,05 \mathrm{aA}$ & $-0,34 \mathrm{aA}$ & $-0,19 \mathrm{aA}$ \\
& & $-100 \mathrm{kPa}$ & & \\
SDE & $1,22 \mathrm{aA}$ & $1,12 \mathrm{aA}$ & $1,28 \mathrm{aA}$ & $0,12 \mathrm{aA}$ \\
SD & $1,38 \mathrm{aA}$ & $1,19 \mathrm{aA}$ & $1,21 \mathrm{aA}$ & $0,36 \mathrm{aA}$ \\
SDNF & $1,23 \mathrm{aA}$ & $1,23 \mathrm{aA}$ & $1,28 \mathrm{aA}$ & $0,08 \mathrm{aA}$ \\
\hline SDE & &
\end{tabular}

SDE: semeadura direta escarificada; SD: semeadura direta; SDNF: semeadura direta cultivada com nabo forrageiro. As letras minúsculas comparam médias entre tratamentos em cada época e profundidade; e as maiúsculas, as médias entre os anos dentro de cada tratamento e profundidade. Médias seguidas pela mesma letra não diferem ente si pelo teste Tukey a $5 \%$. 
potencias mátricos: -6, -10 e $-30 \mathrm{kPa}$. A $\mathrm{Ka}$ igual a $1 \mu^{2}(\log K a=0)$ indica ausência de fluxo em razão de os poros estarem bloqueados e não fazendo parte do transporte convectivo do ar, o que acontece quando os poros estão preenchidos com água ou caso não estejam conectados (McQueen e Shepherd, 2002). A ocorrência de poros muito pequenos em solos com alto teor de argila também propicia valores de $K a$ próximos de zero, conforme relatado por Rodrigues et al. (2011). Porém, como era esperado, conforme o potencial mátrico de água no solo foi reduzido houve aumento da $\mathrm{Ka}$ em todos os tratamentos e profundidades, uma vez que o espaço poroso anteriormente ocupado por água passou a ser preenchido com ar.

Embora o SDNF tenha apresentado maior $K a$, somente no potencial mátrico de $-6 \mathrm{kPa}$ para a camada de 0,00-0,05 m, esse fato é de extrema importância, uma vez que a região apresenta alto índice pluviométrico (acima $1.400 \mathrm{~mm}$ ). A ausência de diferença significativa entre os anos para esse tratamento nessa profundidade e nesse potencial indica que mesmo 18 meses após o cultivo do nabo forrageiro houve manutenção da $K a$ no potencial mátrico de $-6 \mathrm{kPa}$. Isso já não ocorreu para o tratamento $\mathrm{SDE}$, indicando que em regiões úmidas o efeito da escarificação mecânica tende a ser mais curto, tendo em vista uma mais rápida reacomodação das partículas, conferindo assim maior resiliência ao solo (Silva et al., 2012; Nunes et al., 2014).

A permeabilidade ao ar foi maior na camada de 0,00-0,05 m, comparada à camada de 0,05-0,10 m. Provavelmente, os maiores valores de $K a$ na camada superficial podem ser atribuídos à menor densidade do solo, comumente apresentada por essa camada em relação à camada de 0,05-0,10 m. Maiores valores de $K a$ na camada superior em comparação à inferior foram também encontrados por Cavalieri et al. (2009), os quais avaliaram a $K a$ em semeadura direta de longa duração em diferentes profundidades, e por Rodrigues et al. (2011), os quais compararam a $K a$ entre preparo convencional e semeadura direta nas profundidades de 0,00-0,10 e 0,10-0,20 m, ambos os trabalhos conduzidos em Latossolo Vermelho na mesma região deste estudo.

$\mathrm{O}$ índice $K_{1}$ obtido pela relação entre $K a / \varepsilon_{\mathrm{a}}$ é considerado por Groenevelt et al. (1984) importante índice para determinar se as diferenças em $K a$ podem ser atribuídas à $\varepsilon_{\mathrm{a}}$ ou a outros aspectos geométricos do espaço poroso, como a distribuição do tamanho de poros, tortuosidade e continuidade. Dessa forma, quanto maior for o valor de $K_{1}$ maior será a capacidade de transporte de ar em razão da continuidade do sistema poroso do solo. $\mathrm{O}$ índice $K_{1}$ também apresentou uma distribuição não normal; sendo assim, seus valores foram transformados para a forma logarítmica com a finalidade de manter o uso de análise estatística paramétrica. Os valores médios de $K_{1}$ para os potenciais estudados estão apresentados na figura 2 .

No potencial mátrico de $-6 \mathrm{kPa}$ na camada de 0,00-0,05 m, o tratamento SDNF apresentou maior continuidade de poros ou menor tortuosidade que os tratamentos SDE e SD. Para os demais potenciais, no entanto, não houve diferenças significativas entre os tratamentos em ambas as épocas avaliadas. $\mathrm{Na}$ comparação entre anos, o $K_{1}$ em SDE e SD nos potenciais mátricos de -6 e $-10 \mathrm{kPa}$ foram significativamente maiores em 2010 que em 2009. Esses resultados são semelhantes aos obtidos para a $K a$. Isso demonstra que o índice de continuidade de poros $K_{1}$ na camada de 0,00-0,05 m foi consistente com os valores de $K a$ e corrobora o fato de que o cultivo do nabo forrageiro pode resultar em melhoria do sistema poroso do solo.

A medida da $K a$ próximo à capacidade de campo é considerada a ideal por permitir uma estimativa adequada da continuidade da fração de poros grandes (Iversen et al., 2003). No entanto, os resultados para $K a$ e $K_{1}$ no potencial mátrico de $-10 \mathrm{kPa}$ não evidenciaram as possíveis diferenças existentes entre os tratamentos. Os resultados para $K a$ e $K_{1}$ indicaram o potencial mátrico de - $6 \mathrm{kPa}$ na camada de 0,00-0,05 m como mais adequado na diferenciação dos manejos aplicados ao solo. Isso pode ser explicado pelo fato de que em menores potenciais $(-10,-30 \mathrm{e}-100 \mathrm{kPa})$ as maiores condições de aeração dificultam a diferenciação dos tratamentos, quando comparados a condições de maior umidade.

Na camada de 0,05-0,10 m, no potencial mátrico de $-30 \mathrm{kPa}$, o tratamento $\mathrm{SDE}$ apresentou $K_{1}$ significativamente maior que o SD e SDNF em 2009. Isso evidencia que aos seis meses após a escarificação mecânica houve maior continuidade de poros do que em SD e SDNF. A ausência de diferença estatística entre épocas para o SDE no potencial de $-30 \mathrm{kPa}$ indica que a continuidade de poros foi mantida até os 18 meses após escarificação; entretanto, para a segunda época não houve diferenças entre os tratamentos (Figura 3). Esse resultado corrobora os de Nunes et al. (2014), os quais também verificaram melhoria de propriedades físicas do solo até 18 meses, após escarificação mecânica em um Nitossolo Vermelho de textura argilosa. No entanto, divergente dos relatados por Nicoloso et al. (2008) e Silva et al. (2012), os quais observaram efeito de curta duração para a escarificação mecânica, respectivamente, de nove e seis meses. Todavia, esses trabalhos não avaliaram a permeabilidade ao ar, nem os índices a ela relacionados. Sendo o $K 1 \mathrm{um}$ índice que permite inferências de aspectos geométricos do espaço poroso do solo, como distribuição de tamanho, tortuosidade e continuidade de poros, infere-se que esse foi um parâmetro sensível em detectar a persistência da escarificação mecânica do solo após 18 meses de sua aplicação. Os outros potenciais não apresentaram 

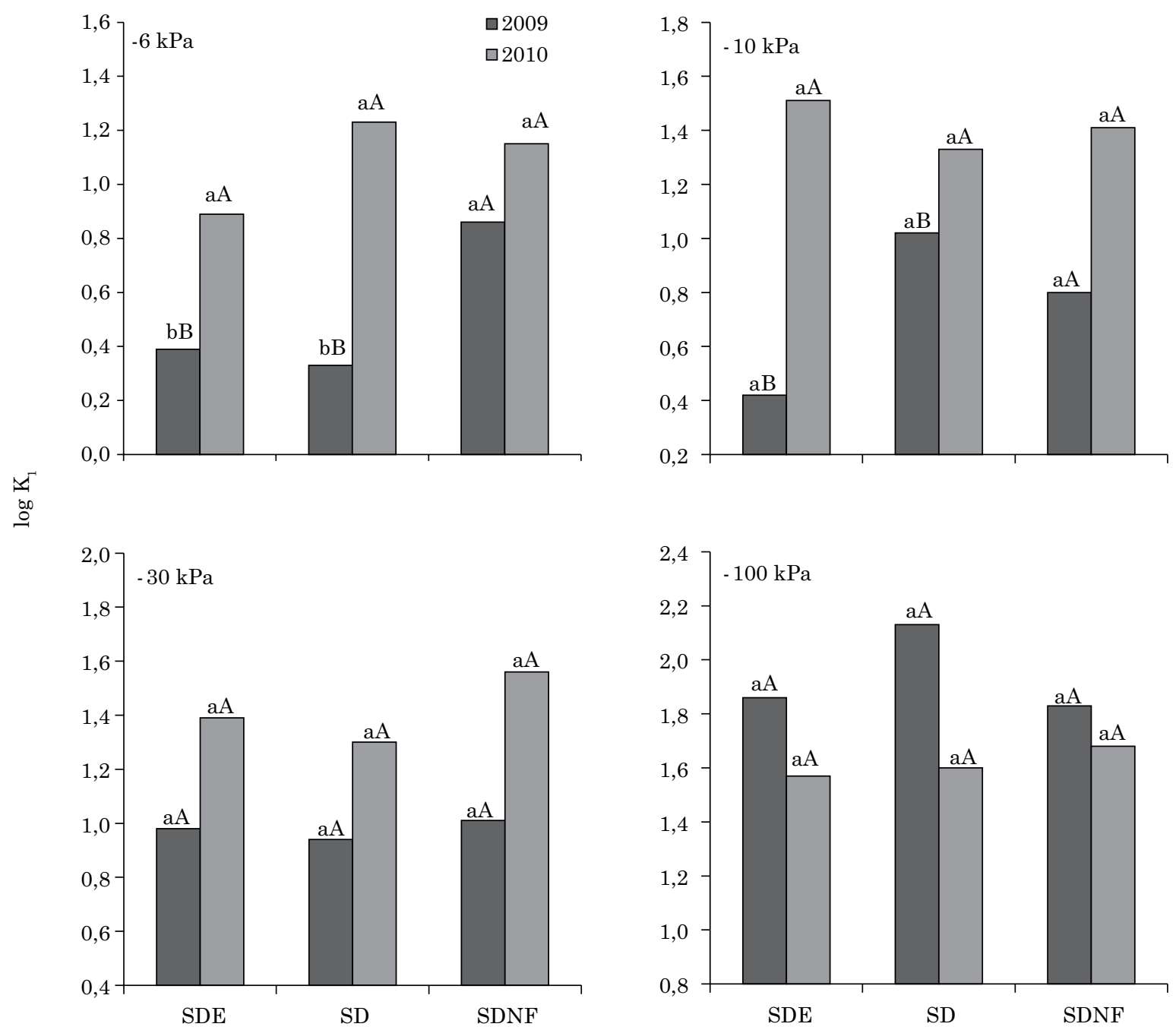

Figura 2. Valores médios de $\log K_{1}$ (índice de continuidade, $K_{1}$ ) para a camada de 0,00-0,05 m em diferentes potenciais mátricos, para a cama de semeadura do milho (2009) e da soja (2010) com semeadura direta escarificada (SDE), semeadura direta (SD) e semeadura direta cultivada com nabo forrageiro (SDNF). Barras seguidas de mesma letra minúscula indicam que as médias dos tratamentos não diferem em cada ano e as seguidas de mesma letra maiúscula indicam que as médias entre os anos dentro de cada tratamento não diferiram pelo teste Tukey a $5 \%$.

diferenças estatísticas entre tratamentos e nem entre épocas, com exceção do potencial mátrico de $-100 \mathrm{kPa}$, em que todos os tratamentos foram estatisticamente maiores em 2009, comparado a 2010. Essa maior continuidade de poros em $-100 \mathrm{kPa}$ foi considerada um resultado inesperado, tendo em vista que nesse potencial dificilmente os solos apresentariam problemas de aeração, sendo visto realmente como uma exceção.

A permeabilidade ao ar foi relacionada com a porosidade de aeração pelo modelo linearizado da equação 4, obtendo os valores dos parâmetros $\mathrm{M}$ e N. A inclinação da reta entre $\log K a$ e $\log \varepsilon_{\mathrm{a}}$ que é o parâmetro $\mathrm{N}$ é um índice de continuidade de poros que representa a porcentagem de poros contínuos interligados disponíveis para o fluxo de ar com o aumento da $\varepsilon_{\mathrm{a}}$. Os valores médios de $\mathrm{N}$ não diferiram entre tratamentos e entre épocas para ambas as profundidades avaliadas (Figura 4). Esse resultado demonstra que, pela avaliação do índice $\mathrm{N}$, todos os tratamentos em ambas as épocas e profundidades apresentaram continuidade de poros semelhantes. De maneira geral, a camada de 0,00-0,05 $\mathrm{m}$ evidenciou maiores valores de $\mathrm{N}$, que variaram de 1,91 a 3,73 , do que a camada de $0,05-0,10 \mathrm{~cm}$, que teve o $\mathrm{N}$ variando de 0,89 a 2,47, indicando que a camada de 0,00-0,05 m revelou melhor continuidade de poros ou menor tortuosidade que a camada de 0,05-0,10 m. Valores semelhantes do índice $\mathrm{N}$ foram encontrados por Silveira Junior et al. (2013) para o mesmo experimento e por Rodrigues et al. (2011) para um sistema semeadura direta em Latossolo Vermelho 

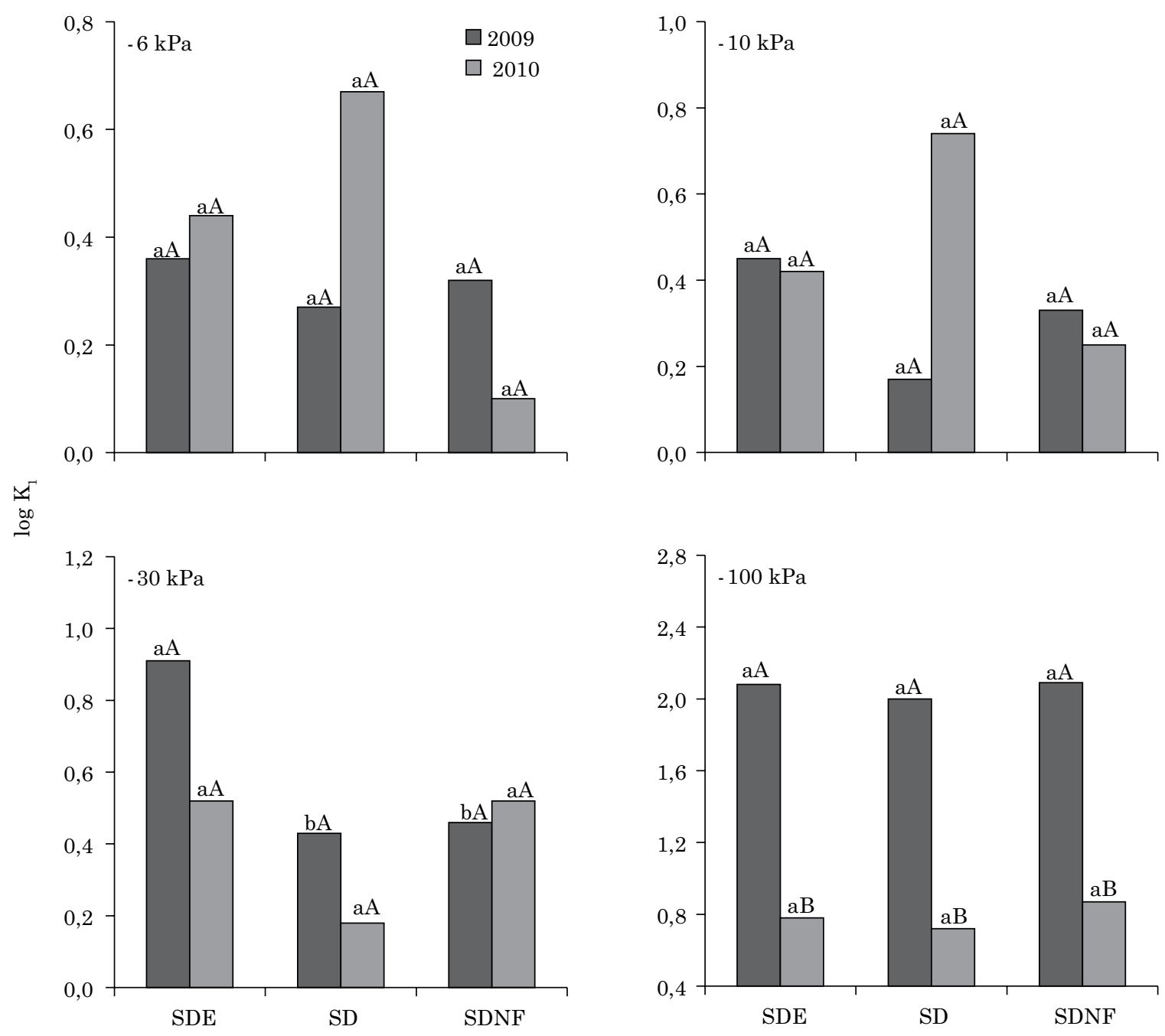

Figura 3. Valores médios de $\log K_{1}$ (índice de continuidade de poros, $K_{1}$ ) para a camada de 0,05-0,10 m com diferentes potenciais mátricos para a cama de semeadura do milho (2009) e da soja (2010) com semeadura direta escarificada (SDE), semeadura direta (SD) e semeadura direta cultivada com nabo forrageiro (SDNF). Barras seguidas de mesma letra minúscula indicam que as médias dos tratamentos não diferem em cada ano e as seguidas de mesma letra maiúscula indicam que as médias entre os anos dentro de cada tratamento não diferiram pelo teste Tukey a $5 \%$.

na mesma região do estudo. No entanto, Ball et al. (1988) encontraram valores superiores de $\mathrm{N}$ $(3,87$ a 8,64$)$ para a camada superficial em sistema semeadura direta. Para esses autores, quanto maior o valor de $\mathrm{N}$ maior será a taxa de abertura de caminhos de poros contínuos com o aumento da porosidade de aeração.

A porosidade bloqueada representa o valor de porosidade abaixo do qual o fluxo de ar através do solo cessa por causa da descontinuidade do sistema poroso desse solo (Poulsen et al., 2007). Os valores médios de porosidade bloqueada não diferiram significativamente entre tratamentos nem entre épocas para ambas as profundidades (Figura 5), indicando que a porosidade bloqueada seguiu tendência similar aos resultados do índice $\mathrm{N}$.
Na primeira época, o volume de poros bloqueados foi semelhante entre profundidades em cada tratamento. Já na segunda, houve aumento no volume de poros bloqueados da camada de 0,00-0,05 para 0,05-0,10 m no SDE e SDNF. Todavia, essas diferenças não foram significativas. Para a primeira época, a porosidade bloqueada variou de 12,55 a $16,96 \%$, enquanto, na segunda, essa variação foi de 13,30 a $22,23 \%$.

A porosidade de aeração não diferiu estatisticamente entre tratamentos, nem entre épocas em ambas as profundidades na capacidade de campo $(-10 \mathrm{kPa})$ (Figura 6). A linha tracejada na figura 6 representa o limite mínimo de $10 \%$ de porosidade de aeração para desenvolvimento das plantas (Grable e Siemer, 1968). Dessa forma, somente o tratamento SD na camada 

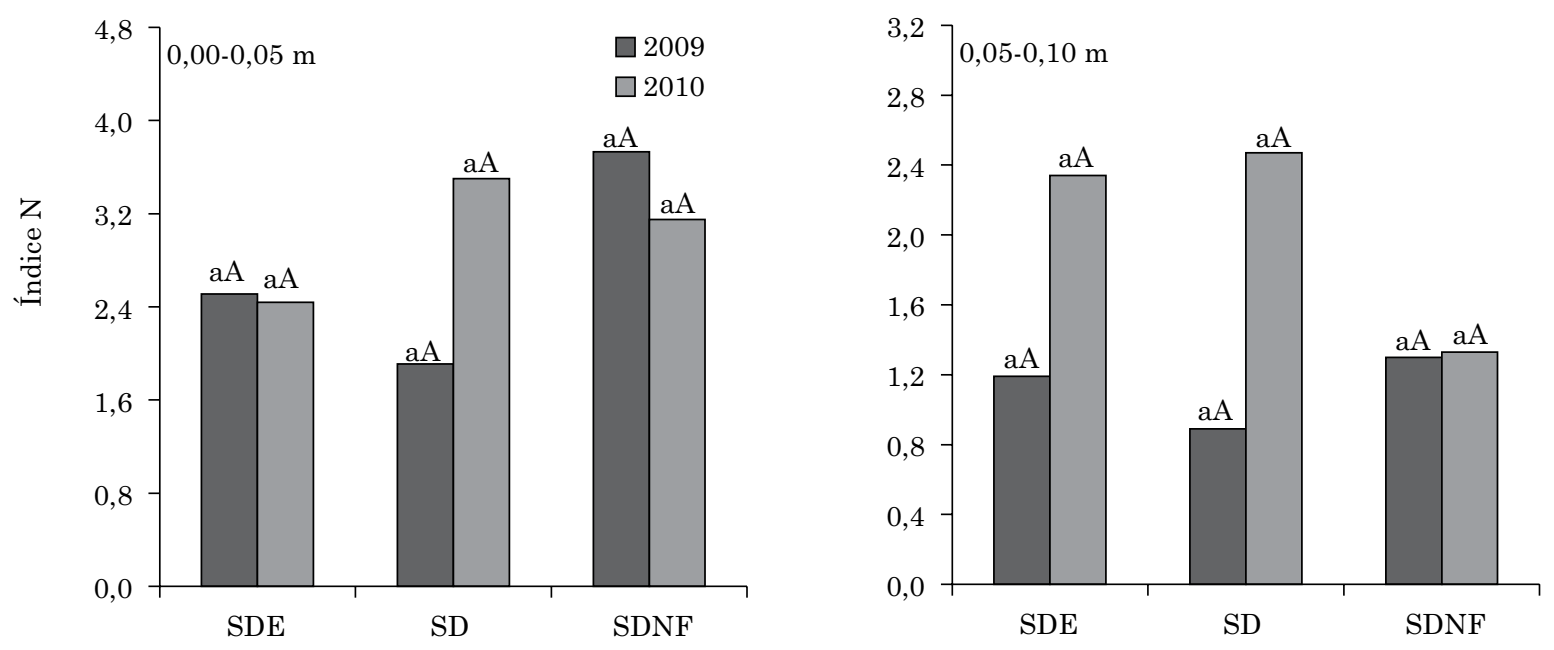

Figura 4. Valores médios do parâmetro $\mathrm{N}$ obtidos do modelo: $\log K_{a}=\log \mathrm{M}+\mathrm{N} \log \varepsilon_{\mathrm{a}}$ para os tratamentos semeadura direta escarificada (SDE), semeadura direta (SD) e semeadura direta cultivada com nabo forrageiro (SDNF) nas camadas 0,00-0,05 e 0,05-0,10 m na cama de semeadura do milho (2009) e da soja (2010). Barras seguidas de mesma letra minúscula indicam que as médias entre tratamentos em cada ano para cada profundidade não diferem pelo teste Tukey a $5 \%$, e seguidas de mesma letra maiúscula as médias entre os anos para cada tratamento e cada profundidade não diferem pelo teste Tukey a $5 \%$.
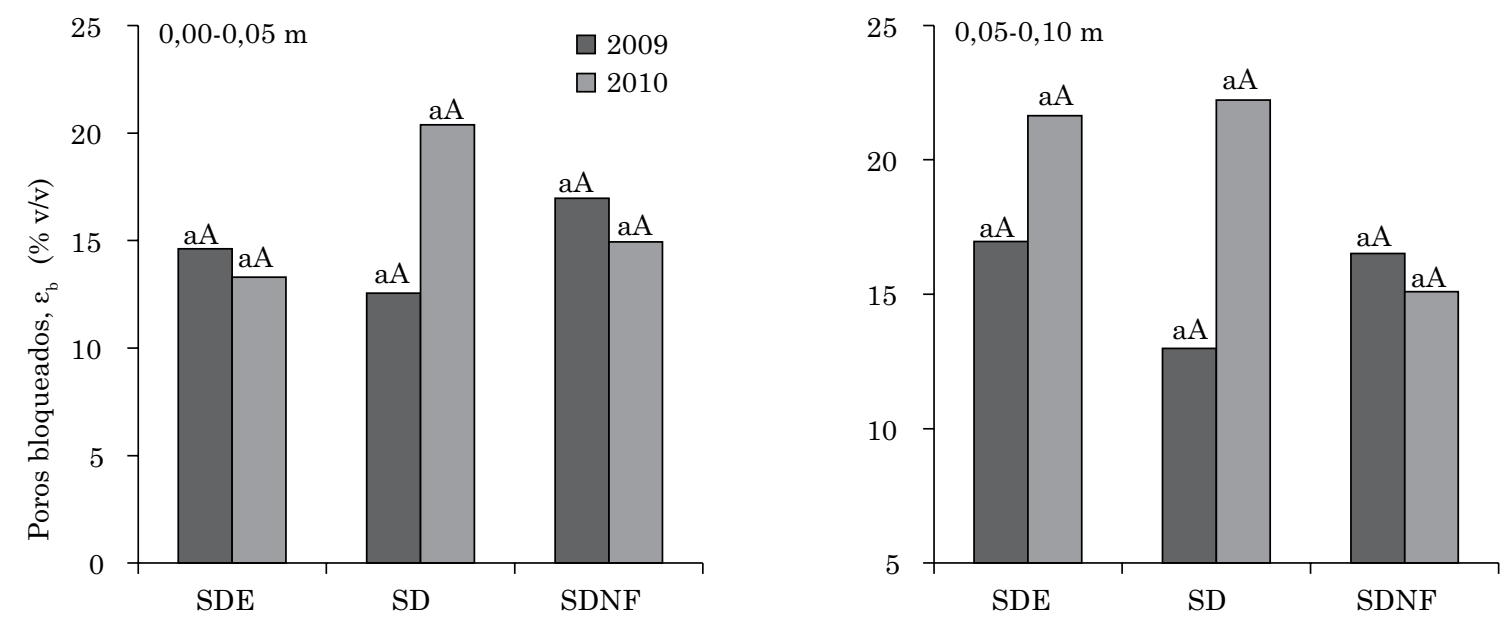

Figura 5. Valores médios da porosidade bloqueada $\left(10^{-\log (M) / N}\right)$ para os tratamentos semeadura direta escarificada (SDE), semeadura direta (SD) e semeadura direta cultivada com nabo forrageiro (SDNF) nas camadas 0,00-0,05 e 0,05-0,10 m na cama de semeadura do milho (2009) e da soja (2010). Barras seguidas de mesma letra minúscula indicam que as médias entre tratamentos em cada ano para cada profundidade não diferem pelo teste Tukey a $5 \%$, e seguidas de mesma letra maiúscula as médias entre os anos para cada tratamento e cada profundidade não diferem pelo teste Tukey a $5 \%$.

de 0,05-0,10 m em 2009 apresentou porosidade de aeração menor que o limite mínimo, com cerca de $7 \%$. Valor semelhante de porosidade de aeração também para o sistema semeadura direta nessa camada foi obtido por Lipiec et al. (2006). Mesmo com o limite mínimo de aeração de 10 \%, Schjønning et al. (1999) destacaram que em porosidade de aeração menor que esse valor pode ocorrer significante difusividade de gás, especialmente em solos bem estruturados, que é o caso dos solos sob semeadura direta. No entanto, em 2010, o valor da porosidade de aeração no SD aumentou para $19 \%$. Todavia, observou-se que tanto SDE quanto SDNF também apresentaram porosidade de aeração próxima do limite de $10 \%$, que foi de $11 \%$ para o SDE e $12 \%$ para o SDNF, na camada de 0,05-0,10 m. 

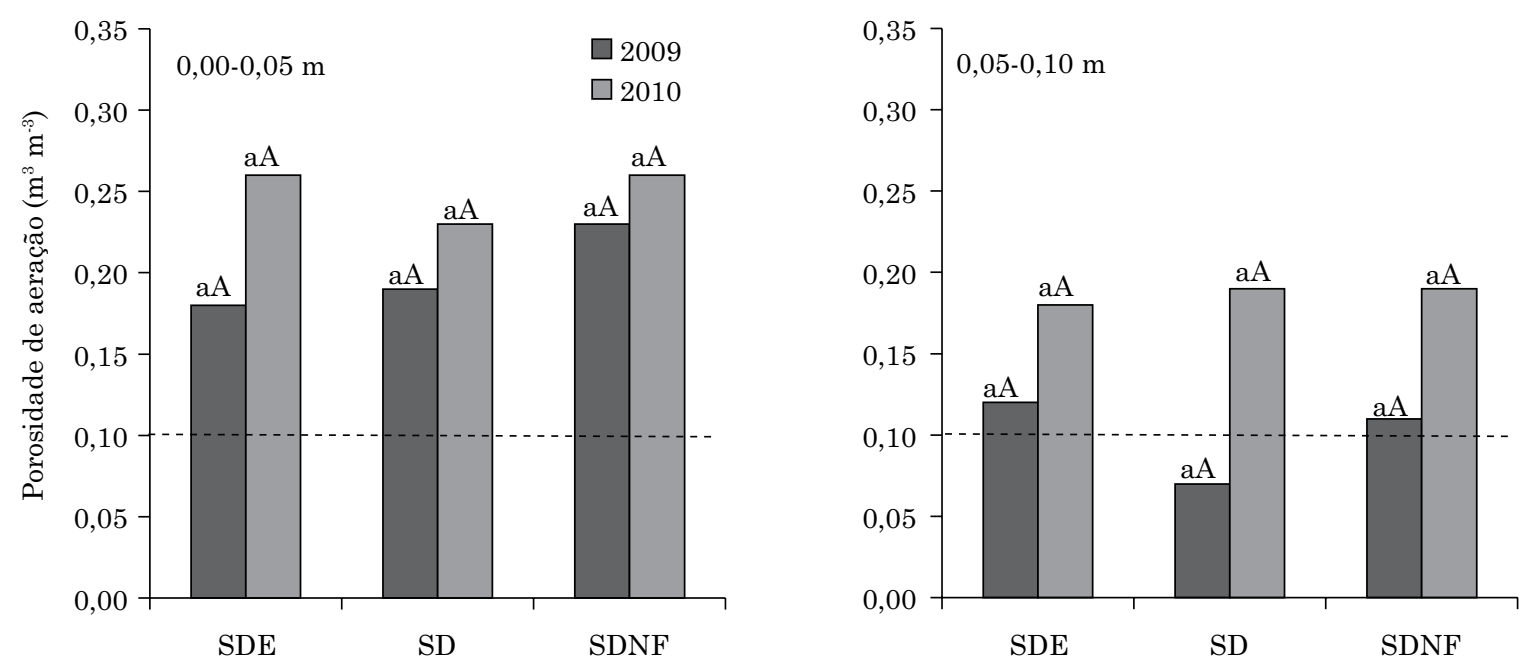

Figura 6. Valores médios da porosidade de aeração $\left(\varepsilon_{a}\right)$ na capacidade de campo (-10 kPa) para os tratamentos semeadura direta escarificada (SDE), semeadura direta (SD) e semeadura direta cultivada com nabo forrageiro (SDNF) nas camadas 0,00-0,05 e 0,05-0,10 m para a cama de semeadura do milho (2009) e da soja (2010). Barras seguidas de mesma letra minúscula indicam que as médias entre tratamentos em cada ano para cada profundidade não diferem pelo teste Tukey a $5 \%$, e seguidas de mesma letra maiúscula as médias entre os anos para cada tratamento e cada profundidade não diferem pelo teste Tukey a $5 \%$.

\section{CONCLUSÕES}

Os efeitos benéficos da escarificação biológica na permeabilidade do solo ao ar e no índice de continuidade de poros $K 1$ persistiram aos 18 meses, após sua aplicação.

A escarificação mecânica resultou em maior continuidade do sistema poroso do solo, avaliado pelo índice $K 1$, com persistência desse efeito aos 18 meses após sua aplicação.

$\mathrm{O}$ índice $\mathrm{N}$, a porosidade bloqueada e a porosidade de aeração não se evidenciaram sensíveis à escarificação mecânica e à biológica do solo.

\section{REFERÊNCIAS}

Abreu SL, Reichert JM, Reinert DJ. Escarificação mecânica e biológica para a redução da compactação em Argissolo franco arenoso em plantio direto. R Bras Ci Solo. 2004;28:519-31.

Ahuja LR, Naney JW, Green RE, Nielsen DR. Macroporosity to characterize spatial variability of hydraulic conductivity and effects of land management. Soil Sci Soc Am J. 1984;48:699-702.

Angers DA, Caron J. Plant-induced changes in soil structure: Processes and feedbacks. Biogeochemistry. 1998;42:55-72.

Atkinson BS, Sparkes DL, Mooney SJ. The impact of soil structure on the establishment of winter wheat (Triticum aestivum). Eur J Agron. 2009;30:243-57.

Atkinson BS, Sparkes DL, Mooney SJ. Using selected soil physical properties of seedbeds to predict crop establishment. Soil Till Res. 2007;97:218-28.
Ball BC, O'sullivan MF, Hunter R. Gas diffusion, fluid flow and derived pore continuity indices in relation to vehicle traffic and tillage. J Soil Sci. 1988;39:327-39.

Braunack WA, Dexter AR. Soil aggregation in the seedbed: A review. II. Effect of aggregate sizes on plant growth. Soil Till Res. 1989;14:281-98.

Calonego JC, Borghi E, Crusciol CA. Intervalo hídrico ótimo e compactação do solo em cultivo consorciado de milho e braquiária. R Bras Ci Solo. 2011;35:2183-90.

Câmara RK, Klein VA. Escarificação em plantio direto como técnica de conservação do solo e da água. R Bras Ci Solo. 2005;29:789-96.

Cavalieri KMV, Silva AP, Tormena CA, Leão TP, Dexter AR, Hakansson I. Long-term effects of no-tillage on dynamic soil physical properties in a Rhodic Ferrasol in Parana, Brazil. Soil Till Res. 2009;103:158-64.

Chen G, Weil RR. Penetration of cover crops roots through compacted soil. Plant Soil. 2010. doi 10.1007/s11104-009-0223-7.

Cresswell HP, Kirkegaard JA. Subsoil amelioration by plant root-the process and the evidence. Aust J Soil Res. 1995;33:221-39.

Empresa Brasileira de Pesquisa Agropecuária - Embrapa. Centro Nacional de Pesquisa de Solos. Sistema brasileiro de classificação de solos. $2^{\text {a }}$.ed. Rio de Janeiro: 2006.

Evans SD, Lindstrom MJ, Voorhees WB, Moncrief JF, Nelson GA. Effect of subsoiling and subsequent tillage on soil bulk density, soil moisture, and corn yield. Soil Till Res. 1996;38:35-46.

Federação Brasileira de Plantio Direto na Palha - Febrapdp. [Acessado em 28 jan. 2014]. Disponível em: http://www. febrapdp.org.br.

Figueiredo GC. Avanços metodológicos e instrumentais em física do solo [tese]. Piracicaba: Escola Superior de Agricultura Luiz de Queiroz; 2010. 
Grable AR, Siemer EG. Effects of bulk density, aggregate size, and soil water suction on oxygen diffusion, redox potential and elongation of corn roots. Soil Sci Soc Am J. 1968;32:180-6.

Groenevelt PH, Kay BD, Grant CD. Physical assessment of a soil with respect to rooting potential. Geoderma. 1984;34:101-14.

Guedes Filho O, Silva AP, Giarola NFB, Tormena CA. Structural properties of the soil seedbed submitted to mechanical and biological chiseling under no-tillage. Geoderma. 2013;204/205:94-101.

Håkansson I, Myrbeck A, Etana A. A review of research on seedbed preparation for small grains in Sweden. Soil Till Res. 2002;64:23-40.

Instituto Agronômico do Paraná - Iapar. Cartas climáticas do Paraná: Edição ano 2000, versão 1.0. [CD-ROM]. Londrina: 2000.

Iversen BV, Moldrup P, Schjønning P, Jacobsen OH. Field application of a portable air permeameter to characterize spatial variability in air and water permeability. Vadose Zone J. 2003;2:618-26.

Kubota A, Hoshiba K, Bordon J. Green-manure turnip for soybean based no-tillage farming systems in eastern Paraguay. Sci Agric. 2005;62:150-8.

Lipiec J, Kus J, Nosalewicz A, Turski M. Tillage system effects on stability and sorptivity of soil aggregates. Inter Agrophys. 2006;20:189-93.

McQueen DJ, Shepherd TG. Physical changes and compaction sensitivity of a fine-textured, poorly drained soil (Typic Endoaquept) under varying durations of cropping, Manawatu Region, New Zealand. Soil Till Res. 2002;25:217-30.

Meek BD, Detar WR, Rolph D, Rechel ER, Carter LM. Infiltration rate as affected by an alfalfa and no-till cotton cropping system. Soil Sci Soc Am J. 1990;54:505-8.

Nicoloso RS, Amado TJC, Schneider S, Lanzanova ME, Girardello VC, Bragagnolo J. Eficiência da escarificação mecânica e biológica na melhoria dos atributos físicos de um Latossolo muito argiloso e no incremento do rendimento de soja. R Bras Ci Solo. 2008;32:1723-34.

Nunes MR, Pauletto EA, Denardin JE, Faganello A, Pinto LFS, Scheuneman T. Persistência dos efeitos da escarificação sobre a compactação de Nitossolo sob plantio direto em região subtropical úmida. Pesq Agropec Bras. 2014;49:531-9.

Poulsen TG, Moldrup P, Thorbjørn A, Schjønning P. Predicting air permeability in undisturbed, subsurface sandy soils from airfilled porosity. J Environ Eng. 2007;133:995-1001.

Reichert JM, Suzuki LEAS, Reinert DJ, Horn R, Håkansson I. Reference bulk density and critical degree-of-compactness for no-till crop production in subtropical highly weathered soils. Soil Till Res. 2009:102:242-54.
Reinert DJ, Albuquerque JA, Reichert JM, Aita C, Andrada MMC. Limites críticos de densidade do solo para o crescimento de raízes de plantas de cobertura em Argissolo Vermelho. R Bras Ci Solo. 2008;32:1805-16.

Rodrigues S, Silva AP, Giarola NFB, Rosa JA. Permeabilidade ao ar em Latossolo Vermelho sob diferentes sistemas de manejo. R Bras Ci Solo. 2011;35:105-14.

Roseberg RJ, Mccoy EL. Measurement of soil macropore air permeability. Soil Sci Soc Am J. 1990;54:969-74.

Sas Institute. SAS: User'S guide: Statistics. $9^{\text {th }}$.ed. Cary: 2002.

Secco D, Reinert DJ. Efeitos imediato e residual de escarificadores em Latossolo Vermelho-Escuro sob plantio direto. R Eng Agric. 1997;16:52-61.

Schjønning P, Thomsen IK, Møberg JP, Jonge H, Kristensen $\mathrm{K}$, Christensen BT. Turnover of organic matter in differently textured soils. I. Physical characteristics of structurally disturbed and intact soils. Geoderma. 1999;89:177-98.

Schjønning P, Munkholm LJ, Moldrup P, Jacobsen OH. Modeling soil pore characteristics from measurements of air exchange: the long-term effects of fertilization and crop rotation. Eur J Soil Sci. 2002;53:331-9.

Shapiro SS, Wilk MB. Analysis of variance test for normality (complete samples). Biometrika. 1965;52:591-611.

Silva AP, Leao TP, Tormena CA, Gonçalves ACA. Determination of air permeability in undisturbed soil samples by the decreasing pressure method. R Bras Ci Solo. 2009;33:1535-45.

Silva SGC, Silva AP, Giarola NFB, Tormena CA, Sá JCM. Temporary effect of chiseling on the compaction of a rhodic hapludox under no-tillage. R Bras Ci Solo. 2012;36:547-55.

Silveira Júnior SD, Silva AP, Figueiredo GC, Tormena CA, Giarola NFB. Qualidade física de um Latossolo Vermelho sob plantio direto submetido à descompactação mecânica e biológica. R Bras Ci Solo. 2012;36:1854-67.

Springer DS, Loaiciga HA, Cullen SJ, Everett LG. Air permeability of porous materials under controlled laboratory conditions. Ground Water. 1998;36:558-65.

Unger PW, Kaspar TC. Soil compaction and root growth: A review. Agron J. 1994;86:759-66.

Vieira ML, Klein VA. Propriedades físico-hídricas de um Latossolo Vermelho submetido a diferentes sistemas de manejo. $\mathrm{R}$ Bras $\mathrm{Ci}$ Solo. 2007;31:1271-80

Williams SM, Weil RR. Crop cover root channels may alleviate soil compaction effects on soybean crop. Soil Sci Soc Am J. 2004;68:1403-9. 